\title{
Ares I-X Separation and Reentry Trajectory Analyses
}

\author{
Paul V. Tartabini ${ }^{1}$ and Brett R. Starr ${ }^{1}$ \\ NASA Langley Research Center, Hampton, VA 23681
}

\begin{abstract}
The Ares I-X Flight Test Vehicle was launched on October 28, 2009 and was the first and only test flight of NASA's two-stage Ares I launch vehicle design. The launch was successful and the flight test met all of its primary and secondary objectives. This paper discusses the stage separation and reentry trajectory analysis that was performed in support of the Ares I$X$ test flight. Pre-flight analyses were conducted to assess the risk of stage recontact during separation, to evaluate the first stage flight dynamics during reentry, and to define the range safety impact ellipses of both stages. The results of these pre-flight analyses were compared with available flight data. On-board video taken during flight showed that the flight test vehicle successfully separated without any recontact. Reconstructed trajectory data also showed that first stage flight dynamics were well characterized by pre-flight Monte Carlo results. In addition, comparisons with flight data indicated that the complex interference aerodynamic models employed in the reentry simulation were effective in capturing the flight dynamics during separation. Finally, the splash-down locations of both stages were well within predicted impact ellipses.
\end{abstract}

\section{Nomenclature}

$\mathrm{t}=$ time since separation, $\mathrm{s}$

$\alpha_{\text {Total }}=$ total angle-of-attack, deg

$\mathrm{Xcg}=$ axial location of center of gravity, $\%$ of body length

$\mathrm{Xcp}=$ axial location of aerodynamic center of pressure, $\%$ of body length

\section{Introduction}

$\mathrm{O}$ N October 28, 2009, NASA successfully launched the Ares I-X Flight Test Vehicle (FTV) from pad 39B at the Kennedy Space Center (Fig. 1). Ares I-X was the first and only test flight of the two-stage Ares I Crew Launch Vehicle design.

The main objectives of the flight test were to demonstrate controllability of the Ares I rocket design up to stage separation, successfully separate the first stage booster from the upper stage without recontact, and recover the booster after splash down. To achieve these goals in a timely manner, the Ares I-X FTV employed an existing Space Shuttle Program four-segment reusable solid rocket motor (RSRM) first stage (FS) and an inert Ares I Upper Stage Simulator (USS). An inert fifth segment was added to the foursegment FTV booster to maintain the same OML as the Ares I design, which employed a to-be-designed five-segment solid rocket motor. In addition, both the FS and USS of the Ares I-X FTV were ballasted in order to maintain similarity to Ares I mass properties and to ensure that its suborbital trajectory matched, to the greatest extent possible, the Mach and dynamic pressure profiles of the Ares I first stage design trajectory. ${ }^{1}$ The main elements of the Ares I-X launch vehicle are shown in Fig. 2.

Staging of the Ares I-X Flight Test Vehicle occurred $122.9 \mathrm{~s}$ into the flight at an altitude of $128,640 \mathrm{ft}$. After separation, both the FS and USS descended uncontrolled. The FS was equipped with a recovery system and began a parachute deployment sequence at approximately 15,000 ft altitude

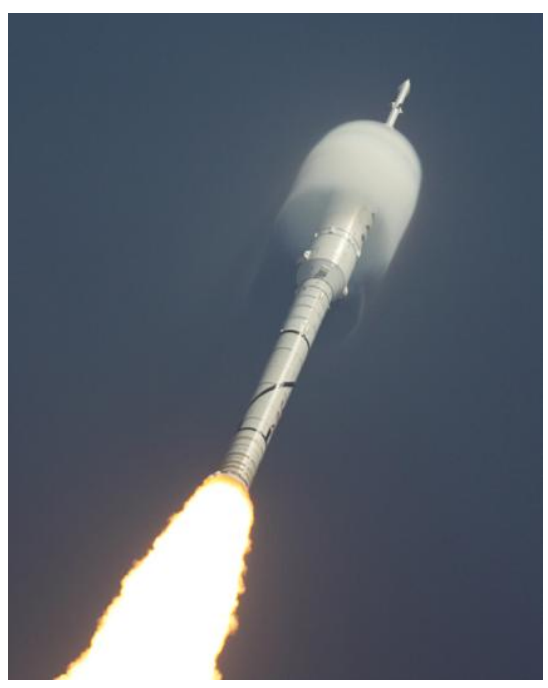

Figure 1. Launch of Ares I-X Flight Test Vehicle on Oct. 28, 2009.

\footnotetext{
${ }^{1}$ Aerospace Engineer, Vehicle Analysis Branch, 1 North Dryden Street, Mail Stop 451, Member.
}

1

American Institute of Aeronautics and Astronautics 
to limit splashdown velocity. The USS descended uncontrolled until water impact and was not recovered.

Pre-flight simulation analyses were conducted to predict the six degree-of-freedom motion of both the FS and USS from the start of separation until splash down and were used to determine the tumbling reentry flight dynamics and the impact footprints for each stage. This paper discusses the development of the Ares I-X separation and reentry simulation and how it was used to verify performance requirements prior to flight. Simulation results were used to understand and quantify the risk of inadvertent recontact between vehicle stages during separation, to predict the flight dynamics and dynamic pressure levels during reentry and to determine the range of expected landing locations of each stage at the time of water impact. This paper also compares preflight results to video and trajectory reconstruction data obtained during flight.

\section{Pre-Flight Simulation Analysis}

\section{A. Separation and Reentry Timeline}

Staging of the Ares I-X Flight Test Vehicle occurred near Mach 4.6 at an altitude of $128,640 \mathrm{ft}$ and a dynamic pressure of $101 \mathrm{psf}$. The Ares I-X separation plane was located at the base of the frustum. The frustum is shown in Fig. 2, on top of the forward skirt extension. At staging, eight booster deceleration motors (BDMs), located on the FS aft skirt, were ignited to reduce the velocity of the FS relative to the USS. During this time the FS moved primarily in an axial direction relative to the USS. Three seconds after staging, four booster tumble motors (BTMs), also located on the aft skirt of the FS, were ignited to induce a tumbling motion predominantly about the negative yaw-axis in order to increase drag and reduce reentry dynamic pressure. Both the BDMs and BTMs had short $(\sim 3$ second) burn times, and the FS subsequently descended, unpowered and uncontrolled, until parachute deployment.

For the first 15-20 seconds after staging, the FS was in the wake of the USS and thus was subjected to aerodynamic interference effects. The FS reached an apogee altitude of approximately 149,000 feet nearly 40 seconds after staging and a maximum dynamic pressure (max-q) of 1,060 psf roughly 130 seconds after staging.

Once the FS descended to an altitude of approximately 15,000 ft, the FS nose cap was jettisoned, releasing a pilot parachute that deployed the drogue parachute. The drogue parachute reached full inflation, decelerating the FS and orienting it into a tail-first attitude. The main parachutes were deployed as the forward skirt extension (FSE) was separated; however, once deployed, two of the three main parachutes experienced failures, resulting in a higherthan-nominal water impact velocity. Parachute performance is not discussed in this paper and further information may be found in Reference 2 .

After separation, the USS naturally tumbled during reentry since it was aerodynamically unstable in forward flight. The USS similarly reached an apogee altitude of nearly 149,000 feet approximately 36 seconds after staging and a maximum dynamic pressure of $2,350 \mathrm{psf}$ close to 126 seconds after staging. The USS did not have a parachute and was not recovered.

\section{B. Simulation Description}

A multi-body simulation of the entire Ares I-X separation and reentry sequence was developed using the Program to Optimize Simulated Trajectories II (POST2). ${ }^{3}$ The POST2 reentry simulation modeled the six-degree-offreedom motion of both the FS and USS from BDM ignition until each stage impacted the water. This simulation became an important tool that was used extensively to conduct pre-flight Monte Carlo analyses to assess the risk of stage recontact during separation, to verify that the FS reentry dynamics resulted in chute deployment conditions within the constraints of the recovery system, and to develop Range Safety data, including predicted impact ellipses, that were required for launch approval by the U.S. Air Force 45th Space Wing at Patrick Air Force Base.

Included in the simulation were models of the winds and atmosphere, RSRM tail-off thrust, BDM thrust, BTM thrust, static aerodynamic forces and moments, stage separation and wake aerodynamic interference effects and aerodynamic damping. The effect of the drogue and main parachutes on the FS was also modeled. Unless otherwise noted, the various models implemented in this simulation are detailed in the following sections.

$$
2
$$

American Institute of Aeronautics and Astronautics 


\section{Separation State}

The POST2 reentry simulation was initiated at the point of stage separation, three seconds after the sensed axial acceleration of the booster dropped below $7.3 \mathrm{ft} / \mathrm{s}^{2}$. A nominal separation state (position, velocity, attitude, attitude rate) of the integrated FTV at the start of staging was determined in a separate POST2 simulation of the ascent trajectory and subsequently used to initialize the reentry simulation.

\section{Mass Properties}

At the start of stage separation, the nominal predicted weight of the FS was 252,300 lb and the nominal predicted weight of the USS was 456,100 lb. For each stage, dispersions were applied to the mass, center-of-gravity locations and moment and products of inertia.

The FS mass properties changed slightly over time due to propellant depletion from the RSRM tailoff, BDM and BTM motor burns. In addition, step changes in mass properties were also modeled at trajectory events when various elements were jettisoned (nose cap jettison, forward skirt extension separation and nozzle extension jettison). Conversely, the USS mass properties remained constant for the duration of reentry.

In addition, it was assumed that slag, a by-product of solid rocket motor combustion, had not accumulated in the FS during ascent. In general, the amount of slag that accumulates during an RSRM burn is uncertain. Because slag accumulates in the aft part of the FS, ignoring its contribution resulted in a more forward cg location for the FS, thus providing an upper bound on the probability of a nose-first reentry, the least desirable type of reentry due to parachute loading constraints (discussed in more detail later).

\section{Propulsion}

Stage separation occurred during the end of the tailoff of the RSRM, and separation was initiated while the RSRM was still thrusting. An RSRM thrust tailoff profile was included in the reentry simulation to model the effect of residual thrust from the main booster during separation.

Also modeled were the eight BDMs used to decelerate the FS relative to the USS and the four BTMs used to induce the FS tumbling motion. These motors were the same as the heritage booster separation motors used for the Space Shuttle solid rocket motor separation. Each motor had a peak vacuum thrust of roughly 23,000 lbf. The overall burn time including tailoff was $\sim 3 \mathrm{~s}$, but the peak thrust duration was less than $1 \mathrm{~s}$. In Monte Carlo analyses, the effect of dispersions in mounting location, mounting alignment, ignition time and burn rate were modeled for each of these 12 motors.

\section{Aerodynamics}

A complex aerodynamic database constructed from wind tunnel data and computational fluid dynamic calculations was employed in the Ares I-X FTV separation and reentry simulation. ${ }^{4-6}$ The database included sixdegree-of-freedom force and moment coefficients as well as aerodynamic damping derivatives for each stage. The database provided the capability to model vehicle aerodynamics throughout the entire reentry, including immediately after separation, when the FS was in proximity to the USS and aerodynamic interference effects were significant.

The FS aerodynamic database was composed of four different data sets. A basic six degree-of-freedom aerodynamic data set was used throughout the reentry and was derived from a mixture of wind tunnel testing and Shuttle RSRM heritage. The basic data included force and moment coefficients that were a function of Mach, total angle-of-attack and aerodynamic roll angle. In addition, yaw, pitch and roll damping derivatives derived from Shuttle RSRM data were also included for the entire reentry simulation.

During the first three seconds of the reentry trajectory (from BDM ignition until BTM ignition), FS stage separation increments were added to the basic force and moment coefficients to model the effect of aerodynamic interaction with the USS. The separation aerodynamics were based on wind tunnel data and the coefficient increments were dependent upon the USS angle-of-attack and the relative distance and orientation between the two stages.

When the BTMs were ignited (three seconds after separation) the FS stage separation increments were replaced by wake aerodynamic increments. These force and moment coefficient increments were added to the axial, normal and pitch moment coefficients to model the effect of the USS wake on the aerodynamic behavior of the FS. The wake aerodynamic increments were based on CFD calculations and were a function of the relative distance and orientation of the FS with respect to the USS. These wake increments were added to the basic aerodynamic coefficients until the database boundaries were exceeded (Axial separation of 100 FS diameters or Radial Separation of 30 FS diameters).

The USS aerodynamic database was composed of three different data sets. Similar to the FS, the USS employed a basic aerodynamic database that was used for the entire reentry and included the six-degree-of-freedom force and moment coefficients as a function of Mach, total angle-of-attack and aerodynamic roll angle, as well as yaw and pitch damping derivatives that were estimated using the engineering code, Aerodynamic Preliminary Analysis System II (APAS). ${ }^{7}$ In addition, wind tunnel-derived stage separation increments were added to the basic 
aerodynamic coefficients during the first three seconds of separation. After three seconds, the USS was assumed to be free of aerodynamic interference effects and only the basic data set was used.

\section{First Stage Parachute Models}

The POST2 Reentry simulation included the effects of the drogue parachute and main parachutes during the final descent phase of the FS. The simulation modeled the reefing and nonlinear inflation of the parachutes, as well as the jettison of the nose cap, forward skirt extension and nozzle extension that occurred at various times throughout the parachute sequence.

\section{Atmosphere and Winds}

Atmosphere and winds were modeled using the Kennedy Space Center range reference atmosphere included in Version 1.4 of the 2007 Global Reference Atmospheric Model (Gram 2007). ${ }^{8}$ The GRAM 2007 model was also used to generate dispersed wind and atmosphere profiles and was capable of producing profiles specific to each month of the year.

\section{Simulation Analyses}

The POST2 Ares I-X FTV reentry simulation was used to perform a number of pre-flight Monte Carlo analyses that were required for flight approval. Analyses were performed by running 5,000 offnominal cases using dispersions of the type listed in Table 1. Simulations were initiated at dispersed separation states that were obtained from the Monte Carlo results of a separate ascent simulation. Additional dispersions were provided by individual discipline teams as either a nominal value with an interval for an assumed uniform distribution, or as a mean value with a standard deviation for an assumed normal distribution. To preserve consisten-

Table 1. Dispersions used in Ares I-X Separation and Reentry Monte Carlo Analyses

\begin{tabular}{|l|l|}
\hline \multicolumn{1}{|c|}{ Dispersion Type } & \multicolumn{1}{c|}{ Description } \\
\hline Separation State & $\begin{array}{l}\text { 12 state variables (position, velocity, attitude, } \\
\text { attitude rate) obtained from a separate Ascent } \\
\text { Monte Carlo Analysis }\end{array}$ \\
\hline Atmosphere/Winds & GRAM 2007 (Version 1.4) pre-generated tables \\
\hline Mass Properties & $\begin{array}{l}\text { Mass increment, cg position offsets, and } \\
\text { Inertia tensor multipliers }\end{array}$ \\
\hline RSRM Tailoff Thrust & 5,000 dispersed thrust profiles \\
\hline BDM \& BTM & $\begin{array}{l}\text { Propellant mean bulk temperature dispersions, } \\
\text { burn rate increment, ignition time delays, } \\
\text { mounting position and alignment dispersions }\end{array}$ \\
\hline Static Aerodynamics & Force and moment multipliers and increments \\
\hline Aerodynamic Damping & Damping derivative multipliers \\
\hline Parachutes & Deployment altitude of drogue and mains \\
\hline
\end{tabular}
cy with the ascent simulation, the reentry simulation used the same 5,000 FS and USS mass property dispersions, RSRM thrust tail-off profiles and perturbed atmosphere/winds tables used to generate the ascent Monte Carlo cases. The reentry Monte Carlo cases were run according to these prescribed distributions and statistics were computed on the output parameters to assess launch risks, to verify that stage separation and reentry flight requirements were met, and to generate data required for range safety. A description and summary of the key pre-flight analyses that were conducted are described below.

\section{Recontact Risk During Separation}

A separation and recontact analysis was performed to determine the relative position and orientation between the FS and USS and to estimate the probability of stage recontact during near-field and far-field separation.

Prior to staging, the tip of the FS nose cone extended 75 inches into the USS cavity (Fig. 3). Staging was initiated with the firing of the 8 FS BDMs followed by firing of a linear shaped charge to break the joint between the FS and USS. Near-field separation was defined as occurring during the first three seconds of separation, as the FS moved out of and away from the USS. There was an initial clearance of 16.9 inches between the two stages (Fig. 3). This clearance increased as the FS moved away from the USS. Once the axial separation between the two stages reached 75 inches, the FS nose cone was clear of the USS frustum. The near-field analysis continued past

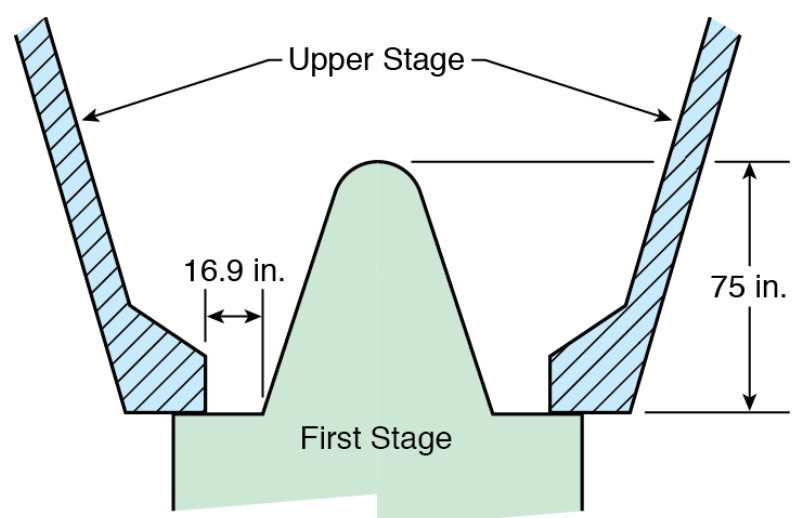

Figure 3. Initial location of stages during separation when near-field proximity is calculated. 
this point until BTM was ignited three seconds after separation, when the FS was typically 2-4 FS diameters away from the USS.

The risk of far-field recontact was considered to begin at BTM ignition, three seconds after staging, and continue until the stages were outside the region of aerodynamic interference and had little or no likelihood of reentering the interference zone. The time at which this point was reached was assumed to be 60 seconds after separation and was based upon relative position statistics determined from Monte Carlo results. Collision detection and proximity analysis was performed for each dispersed Monte Carlo case using a graphical environment tool that integrated time dependent simulation data with high fidelity CAD geometry models. This approach was used to identify cases where far-field recontact had occurred and to compute the closest distance between each stage (proximity distance) from 3 to $60 \mathrm{~s}$ after separation. The schematic in Fig. 4 illustrates the far-field proximity distance metric.

Results are shown in Fig. 5 for the 200 dispersed cases with the smallest minimum proximity distance. Shown in the inset plot is the proximity distance for the first $3 \mathrm{~s}$ of separation, when near-field recontact was a concern. As seen in the inset figure, the FS was clear of the USS by $\sim 1 \mathrm{~s}$ after the initiation of staging, and no cases exhibited near-field recontact. Prior to USS clearance, the relative rotation between the two stages did not exceed $1 \mathrm{deg}$ in any of the dispersed cases.

The results of the far-field proximity analysis are also shown in Fig. 5, where for clarity, the proximity distance is plotted for only the first 30 seconds after separation. The oscillations seen during the early part of separation are due to the tumbling motion of the FS and become less pronounced as the two vehicles move further apart. Out of 5,000 dispersed cases, only eight were identified to have a farfield recontact occurrence (plotted in red in Fig. 5). This result was within the allowable risk

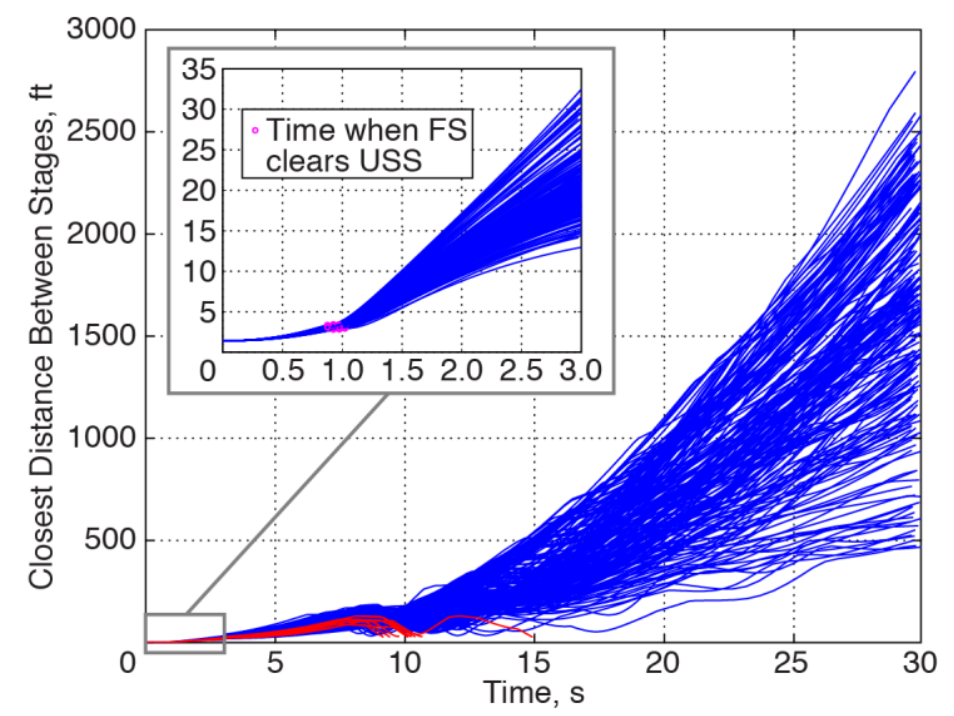

Figure 5. Monte Carlo results showing closest distance between stages. Recontact cases plotted in red. Inset plot magnifies the near-field separation region from $t=0$ to $3 \mathrm{~s}$. tolerance of the Project.

Reentry Flight Dynamics

The Ares I-X POST2 Reentry simulation was used to predict the tumbling flight dynamic behavior of both stages throughout reentry until water impact. For the FS, it was important that the initial tumble rate induced by the BTMs was high enough to inhibit the FS from settling into a nose-first trim attitude during max-q since this type of reentry resulted in the highest dynamic pressure levels and parachute deployment conditions outside the constraints of the recovery system. The attitude of the FS during reentry was also significant in determining where it would impact the water. For the USS, analysis was focused on understanding its flight dynamic behavior immediately after separation when far field recontact was a concern. Because the USS was sufficiently aerodynamically unstable to ensure a tumbling reentry, it did not require tumble motors.

Prior to the flight, the design goal was for the FS to reach max-q conditions at a total angle-of-attack $\left(\alpha_{T}\right)$ between 40 and 140 degrees (referred to as a broad-side reentry). This attitude was desired at max-q because it resulted in low peak dynamic pressure levels and benign parachute deployment conditions. In pre-flight study results, this type of entry was most likely. However, Monte Carlo analysis indicated that for off-nominal cases, even with the initial tumble rate induced by the BTMs, it still was possible for the FS to have a nose-first ( 0 deg $<\alpha_{T}<40$ deg) or 
tail-first (140 deg $<\alpha_{T}<180 \mathrm{deg}$ ) attitude at max-q. A tail-first reentry had higher dynamic pressure levels than a broad-side reentry but still resulted in acceptable parachute deployment conditions.

Analysis indicated that the dispersions with the largest effect on the entry attitude at max-q were the center-of-gravity (cg) and center-ofpressure (cp) locations. For pre-flight analysis, the cg estimates used in the reentry simulation assumed that no slag had accumulated during the ascent solid rocket burn. Because slag accumulates in the tail section of the FS, this assumption placed the nominal FS cg as far forward as it could be, thus providing an upper bound to the likelihood of a nose-first reentry. The uncertainty in the axial cg location was relatively small just prior to flight, on the order of 10". Conversely, the uncertainty in the $\mathrm{cp}$ location was much higher, because of the difficulty in predicting $\mathrm{cp}$ values in tumbling flight conditions.

Monte Carlo results are shown in Fig. 6 where 5,000 off-nominal total angle-of-attack time histories are plotted from separation until parachute deployment. The three entry modes are clearly evident in the region of high dynamic pressure which occurred between $100-150$ seconds after separation. Nose-first reentries were predicted in less than $5 \%$ of the cases. The corresponding dynamic pressure profiles are shown in Fig. 7, where the significantly higher dynamic pressure levels attained during the nose-first reentries is apparent. Both figures show that the nose-first cases reach the max-q point and the parachute deployment conditions sooner than the broad-side and tail-first reentry cases. A tail-first reentry was not as severe as a nose-first reentry due to aerodynamic differences that increased the amount of FS tumbling and exposure to broad-side attitudes where drag was much higher. ${ }^{9}$ A statistical summary of some important parameters at max- $\mathrm{q}$ is listed in Table 2.

For the USS, reentry flight dynamic analysis was focused primarily on the time immediately following stage separation when the two stages were in close proximity to each other. Because pre-flight predictions indicated that the Ares I-X USS would tumble after separation, it was important to evaluate the uncertainty in the USS motion after separation to quantify the risk of far field recontact.

At Mach 4.5, the USS is aerodynamically unstable in forward flight and will immediately

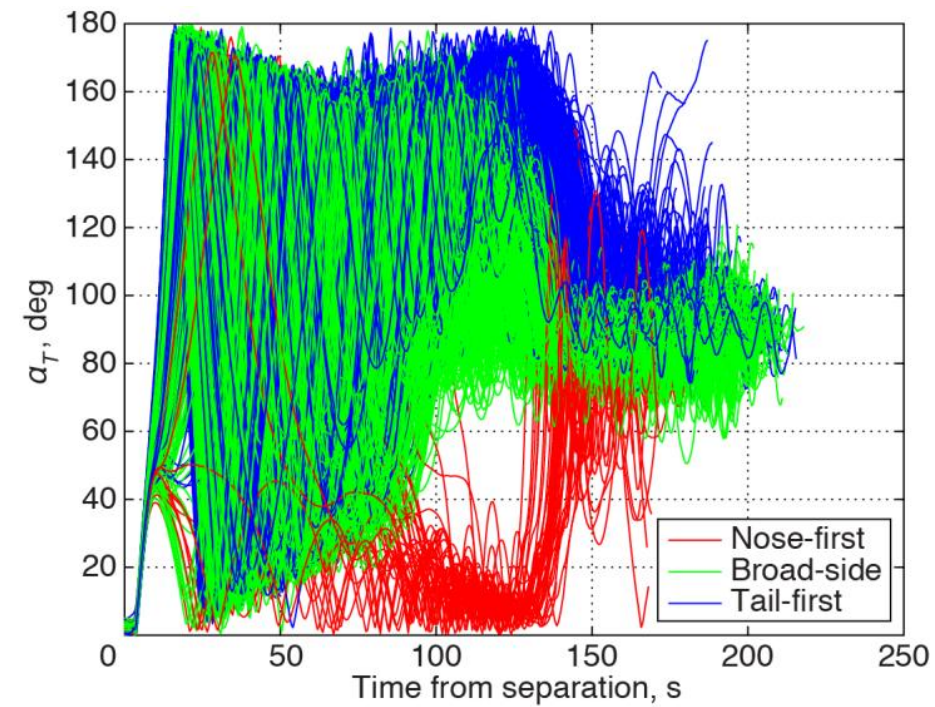

Figure 6. First Stage total angle-of-attack time history, preflight Monte Carlo results.

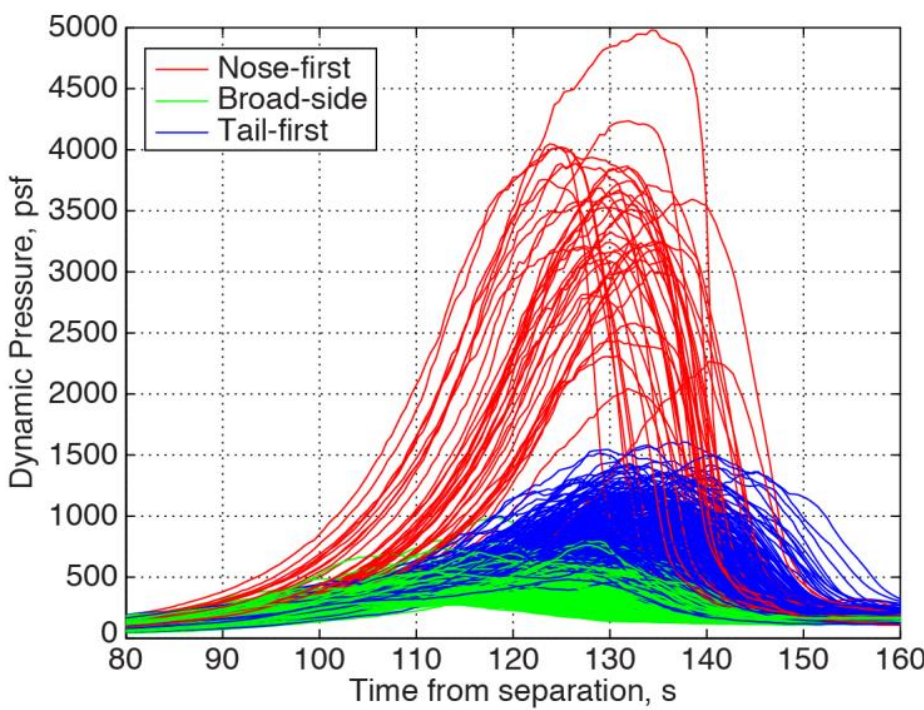

Figure 7. First Stage dynamic pressure and Mach profile, preflight Monte Carlo results.

Table 2. Pre-flight Statistical Results at Maximum Dynamic Pressure

\begin{tabular}{|l|c|c|c|}
\cline { 2 - 4 } \multicolumn{1}{c|}{} & \multicolumn{3}{c|}{ Percentile } \\
\cline { 2 - 4 } \multicolumn{1}{c|}{} & $\mathbf{0 . 1 3}$ & $\mathbf{5 0}$ & $\mathbf{9 9 . 8 7}$ \\
\hline Max q, psf & 291.6 & 573.0 & 4420.4 \\
\hline Alpha Total, deg & 3.6 & 132.6 & 163.8 \\
\hline Mach & 1.71 & 2.47 & 3.62 \\
\hline Altitude, ft & 28850 & 62450 & 86098 \\
\hline Percent Nose-First & 4.1 & & \\
\hline Percent Broad-Side & 58.6 & & \\
\hline Percent Tail-First & 37.3 & \multicolumn{1}{|c}{} \\
\cline { 1 - 2 } & \multicolumn{2}{|c|}{}
\end{tabular}


begin tumbling to a rearward orientation. The significant level of forward-flight instability can be seen in Fig. 8, which shows the USS cg as well as the Mach $4.5 \mathrm{cp}$ location for a range of total angle-of-attack values.

As expected, tumbling was predicted shortly after separation in more than $99 \%$ of pre-flight Monte Carlo cases. Figure 9 shows that this tumbling motion can occur as early as $8 \mathrm{~s}$ after separation, and that by $13 \mathrm{~s}$, roughly half the cases had rotated to a total angle-of-attack value of $120 \mathrm{deg}$. In fact, during flight, the USS did indeed begin tumbling shortly after separation. The postseparation behavior of the USS during flight is discussed in more detail later.

Even though the outer mold line of the USS was designed to be very similar to the Ares I design, upper stage tumbling after separation would not be expected for Ares I for three reasons. First, the cg location of the Ares I Upper Stage will be more forward. To compensate for the dummy fifth segment simulator of the RSRM and other configuration differences, the Ares I-X USS was ballasted so that the overall mass properties of the integrated FS-USS stack during ascent matched the Ares I design. As a result, the added cg location of the USS by itself was further aft than the Ares I Upper Stage. Second, the Ares I stage separation is designed to occur at a dynamic pressure of $\sim 10 \mathrm{psf}$, roughly an order of magnitude lower than for Ares I-X. There was simply not enough impulse in the four-segment RSRM to match the Ares I trajectory beyond Mach 4. Analysis showed that if Ares I-X had separated at the lower Ares I separation dynamic pressure, significantly fewer cases would have tumbled during reentry, and tumbling would not have begun in any case until nearly $40 \mathrm{~s}$ after staging. Finally, in nominal missions, the Ares I liquid upper stage engine will be ignited shortly after separation and will provide thrust vector control.

\section{Landing Ellipses}

A number of pre-flight simulation data prod-

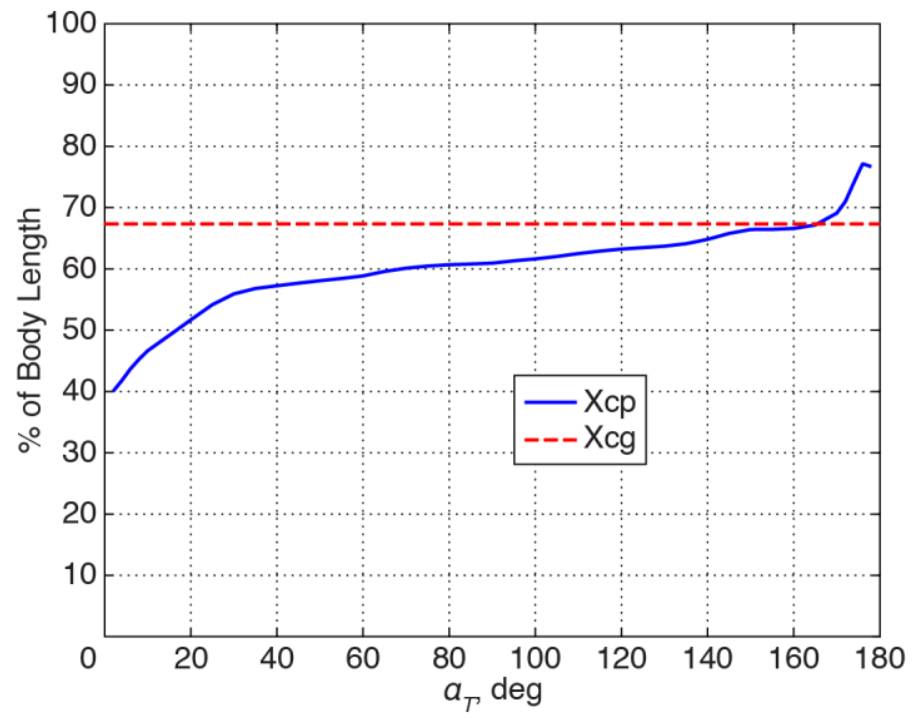

Figure 8. Aerodynamic static stability of USS at separation $($ Mach $=4.5)$.

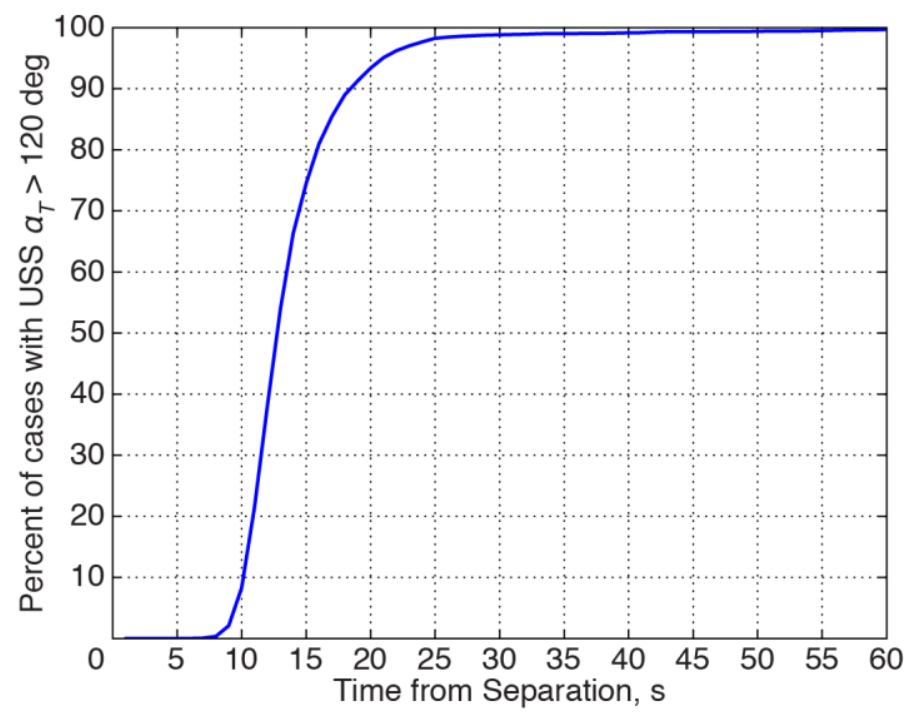

Figure 9. Percentage of pre-flight Monte Carlo cases in which the USS tumbled beyond 120 deg angle-of-attack in a given time from separation.

ucts were assembled into a range safety final flight data package that was delivered to the $45^{\text {th }}$ Space Wing (45SW) prior to launch. ${ }^{10,11}$ These products were necessary to ensure public and launch area safety and were required for launch approval. As part of this package, disposal footprints for each stage were delivered to the $45 \mathrm{SW}$ to provide information on where the spent FS and USS were predicted to land in the water at the end of the test flight. These footprints were delivered as impact ellipses that represented the range of expected locations (latitude/longitude coordinates) of each stage at the time of water impact. These ellipses were used by the $45 \mathrm{SW}$ to establish keepout zones for ship and aircraft traffic and to determine ship placement for first stage tracking and recovery vessels.

Three separate Monte Carlo analyses were performed using the POST2 Ares I-X reentry simulation to determine the FS disposal footprint. The impact ellipse for the FS footprint at splashdown was computed as the ellipse that contained $99.73 \%$ of the latitude/longitude pairs at water impact for 5,000 off-nominal Monte Carlo cases. These trajectories were initiated at separation and modeled all of the major FS reentry events, including BDM firing, BTM firing, aerodynamic interference effects, nose cap jettison, drogue deploy, forward skirt extension separation, main parachute deploy, nozzle extension jettison and splashdown. Next, impact ellipses were determined for the nose cap 
and forward skirt extension, which are jettisoned from the FS during reentry as part of the parachute deployment sequence. An impact ellipse was not generated for the nozzle extension, which is also jettisoned from the FS, because it typically occurs at altitudes below 1000 feet, just 5-10 s before FS splashdown.

The nose cap $(334.4 \mathrm{lbm})$ is jettisoned just prior to drogue chute deploy at an altitude of roughly 15,000 feet. To determine the nose cap impact footprint, a three-degree-of-freedom simulation of the nose cap trajectory was developed. A ballistic coefficient for the nose cap was estimated using Hoerner ${ }^{12}$ drag tables for an approximate nose cap geometry consisting of simple cylindrical and conical shapes. A similar approach was taken for the forward skirt extension (FSE), which separates from the FS as the main parachutes are deployed at a nominal altitude of 5,000 feet. The FSE separates while attached to the drogue parachute (combined mass $=20,295 \mathrm{lbm}$ ). For both of these elements, trajectories were initiated at the point of separation determined in the full FS Monte Carlo analysis and propagated until water impact. The nose cap ballistic coefficient was varied uniformly by $+/-50 \%$, and its disposal footprint was computed as the ellipse that contained $99.73 \%$ of the latitude/longitude pairs at water impact. Similarly, 5,000 FSE trajectories were initiated at FSE separation, and the drogue drag coefficient was varied uniformly by $\pm 2 \%$. The FSE footprint was also computed as the ellipse that contained $99.73 \%$ of the latitude/longitude pairs at water impact. Once the three Monte Carlo analyses were performed, the disposal footprint was determined as the ellipse that enveloped all three (full FS, nose cap and FSE). In fact, there was little difference between the three impact ellipses.

The FS disposal footprint (Fig. 10) was roughly $32.7 \times 9.2 \mathrm{nmi}$ in size (major axis/minor axis) and its centroid was located approximately $121.4 \mathrm{nmi}$ from the launch pad. In Fig. 9 the individual impact locations of the FS from each Monte Carlo case are color coded by entry type. Typically, the broad-side cases populate the left (western) portion of the ellipse and the nose-first and tail-first cases populate the right (eastern) portion.

Due to the tumbling nature of its reentry, it was possible for the USS to break up when flying at broadside attitudes ( $~ 90$ deg angle-of-attack) during the high dynamic pressure regions of reentry. A breakup analysis determined that reentry loads exceeded the lower strength limit of some of the bolted joints connecting the USS segments, but there were no joints where reentry loads exceeded the upper strength limits. Thus, a disposal footprint was determined assuming an intact US throughout reentry. A separate debris analysis was also conducted in which footprints were determined for a range of possible debris configurations. The debris analysis is not discussed here. In fact, video footage from the flight indicated that the USS did not break up during entry and instead splashed down in the water fully intact.

The intact USS disposal footprint was determined in the same manner as the FS disposal footprint, using the POST2 Ares I-X reentry simulation. The impact ellipse was computed as the ellipse that contained $99.73 \%$ of the latitude/longitude pairs at water impact for 5,000 off-nominal Monte Carlo cases. The USS Footprint was roughly $34.2 \times 18.0 \mathrm{nmi}$ in size (major axis/minor axis) and its centroid was located approximately $131.5 \mathrm{nmi}$ from the launch pad. The USS disposal footprint is also plotted in Fig. 10 where it can be seen in relation to the FS footprint.

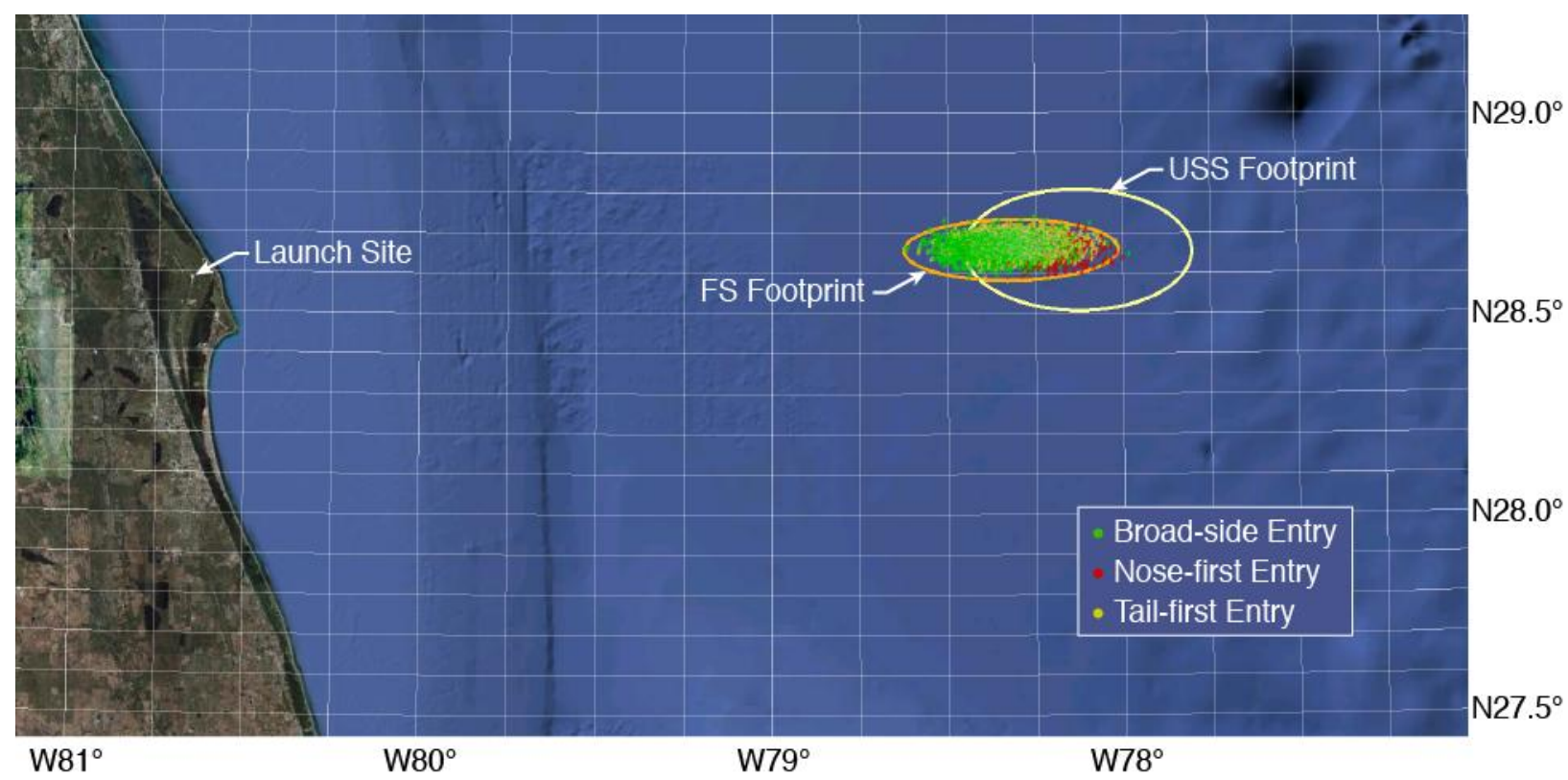

Fig. 10. Disposal footprints for FS (impact locations from pre-flight Monte Carlo results) and USS.

8

American Institute of Aeronautics and Astronautics 
Although the two disposal footprints overlap, Monte Carlo results showed that the USS landed well before the FS in most cases and that the mean distance between impact locations was $\sim 10 \mathrm{nmi}$.

\section{Ares I-X Flight Performance}

The Ares I-X was successfully launched on October 28, 2009, meeting all of its primary and secondary objectives. The separation event was fully successful, and all event sequencing occurred as planned with no near-field or far-field recontact. In addition, separation and reentry flight dynamics were consistent with pre-flight predictions, and both stages landed well within their estimated range safety impact ellipses.

A number of elements in the primary flight test objectives were relevant to separation and reentry. Of particular interest were: 1) Perform an in-flight separation between an Ares I similar first stage and a representative upper stage; and 2) Quantify first stage atmospheric entry dynamics. The following sections highlight the post-flight analysis that was performed to assess whether these separation and reentry test objectives were met. In addition, the impact locations of both stages were determined and compared to the pre-flight disposal footprints.

\section{A. Stage Separation}

The primary goal of the separation maneuver was to demonstrate an Ares I type separation in which stage recontact does not occur.

A Best Estimated Trajectory (BET) for the Ares I-X flight was reconstructed from onboard, ground, and atmospheric measurements. ${ }^{13}$ For the FS, trajectory data was reconstructed from liftoff until splashdown. However, at separation the USS was disconnected from the power supply in the FS, and all sensors on the USS were inoperable. Thus, only radar and atmospheric data were available to reconstruct the USS trajectory. Using this method, the position and velocity of the USS could be estimated, but not the attitude. In addition, the uncertainty in the radar-derived position and velocity was much larger than the corresponding FS flight data. Because of the lack of attitude information and large uncertainties in the USS flight data, it was not possible to determine the separation distance and relative velocity between the stages after separation when near-field and far-field recontact was a concern.

Instead, a qualitative assessment of separation was made, using onboard video from a camera mounted on the FS fifth segment. Frames from this video are shown in Fig. 11 beginning at $t=0 \mathrm{~s}$, the moment of separation, when the BDMs were ignited. From $t=0-3 \mathrm{~s}$ the USS moves axially away from the FS, and there is little or no relative yaw between the stages. At $\mathrm{t}=3 \mathrm{~s}$ the BTMs fire and the FS begins to tumble in yaw, which becomes evident at $\mathrm{t}=4 \mathrm{~s}$. From $\mathrm{t}=0-6 \mathrm{~s}$ no USS rotation can be detected, suggesting that the USS yaw and yaw rate were not affected by the separation or the initiation of the FS tumble. Not until $t=7 \mathrm{~s}$, when there is significant separation distance between the two stages, does any USS yaw rotation become noticeable. This yaw rotation is due to aerodynamic forces. Based on the fact that there was no change in USS attitude after the BTM firing, as well as post-flight inspection of the recovered FS, it was concluded that there was no recontact between the FS and USS during separation.

In some of the video taken from ground-based cameras, it appeared that the USS began to tumble immediately at separation in a positive yaw direction, opposite to the FS tumble direction, giving the impression that its rotation may have been induced by contact with the FS. In fact, this was an artifact from the ground perspective of the camera.

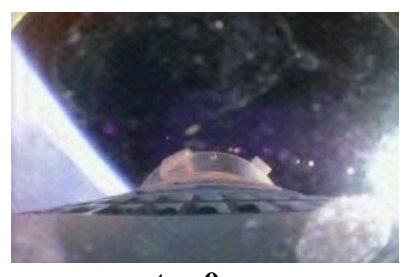

$\mathbf{t}=\mathbf{0} \mathbf{s}$

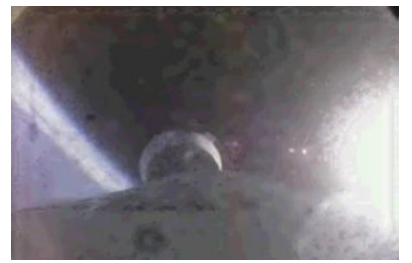

$t=4 \mathrm{~s}$

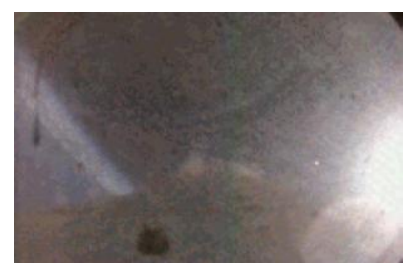

$\mathbf{t}=\mathbf{1} \mathrm{s}$

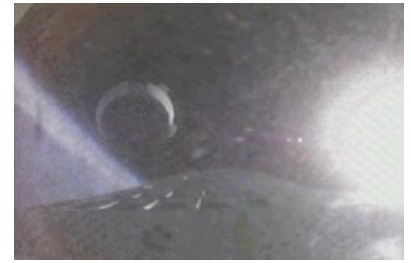

$\mathbf{t}=\mathbf{5} \mathrm{s}$

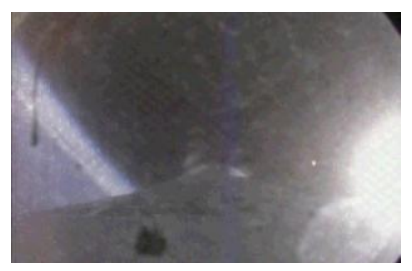

$\mathbf{t}=\mathbf{2} \mathbf{s}$

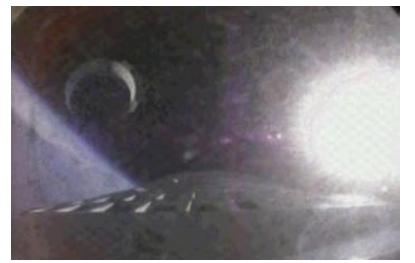

$\mathbf{t}=6 \mathrm{~s}$

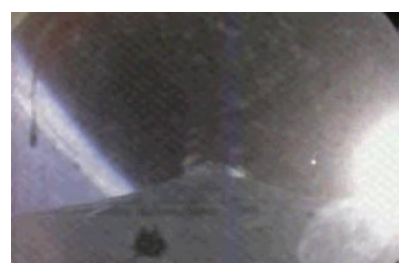

$\mathbf{t}=\mathbf{3} \mathbf{s}$

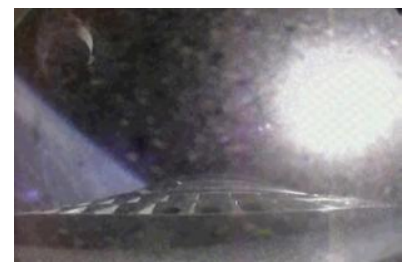

$\mathbf{t}=7 \mathrm{~s}$

Figure 11. Frames from video of Ares I-X stage separation taken by on-board cameras. 

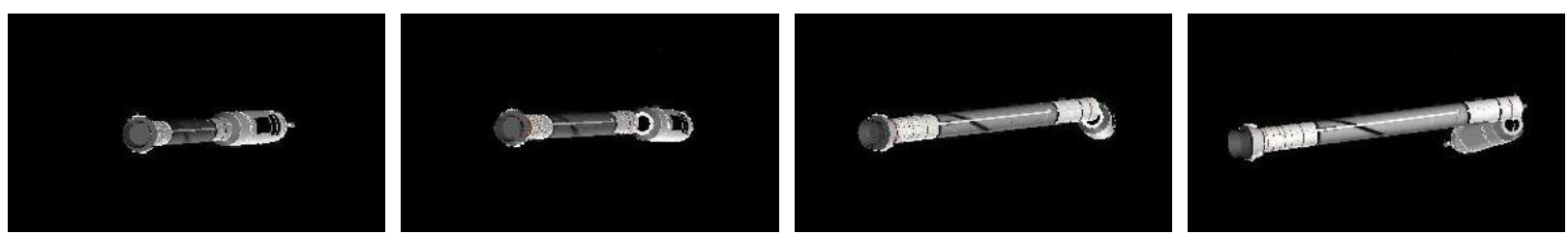

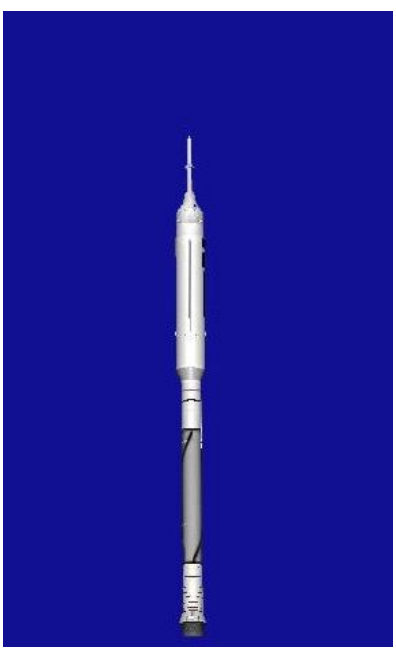

$\mathbf{t}=\mathbf{0} \mathbf{s}$

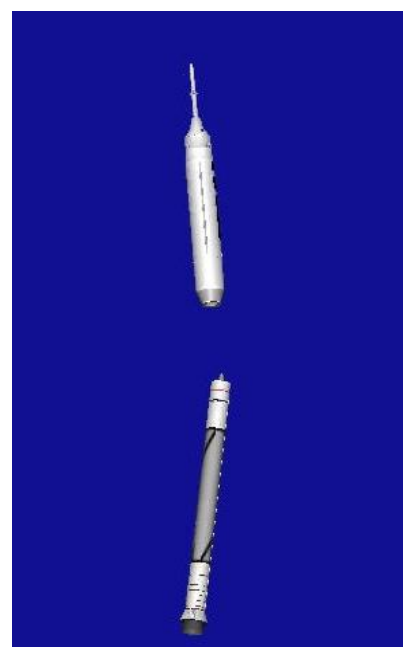

$\mathbf{t}=\mathbf{3} \mathrm{s}$

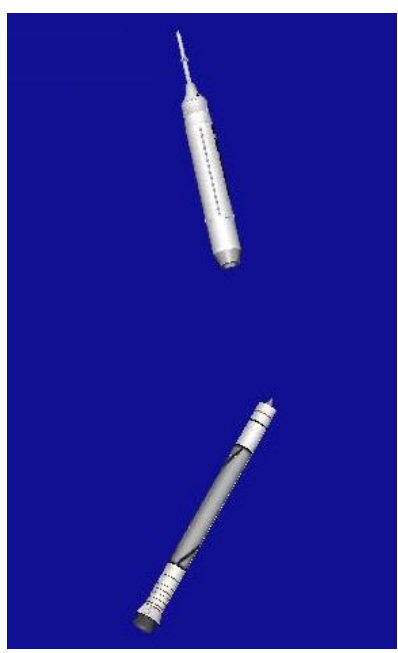

$\mathbf{t}=\mathbf{5} \mathrm{s}$

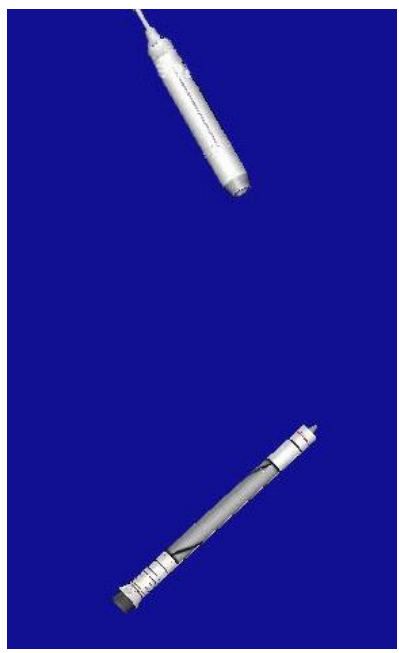

$\mathbf{t}=7 \mathrm{~s}$

Figure 12. Animation of separation simulation started near flight conditions. Top row shows a simulated view from a ground-based camera. Bottom row shows imaginary view above the stages.

Based on pre-flight Monte Carlo results that predicted USS tumbling after separation in more than $99 \%$ of all cases, it was no surprise that the USS began to tumble shortly after separation. Trajectory reconstruction results indicated that separation occurred at $\alpha=-0.42 \mathrm{deg}$ and $\beta=-1.16 \mathrm{deg}$. Due to its aerodynamic instability, it was expected that the USS would begin a positive yaw rotation when starting from a modest, negative sideslip angle. After reviewing the pre-flight Monte Carlo cases, an animation of the separation sequence was created using the case with separation conditions closest to flight. Screen shots from the animation are shown in Fig. 12 from two simulated points of view at selected time points during separation. The top sequence of images mimics the view of the groundbased camera, and the bottom sequence shows the same animation frames from a top view, looking down at the separating stages. While it is difficult to discern how far apart the two stages are when the tumbling motion begins in the ground-based view, the alternate view looking down clearly shows that it was possible for the two stages to tumble as they did without coming into contact with each other.

\section{B. First Stage Reentry Flight Dynamics}

This section compares the FS reentry flight data with the results of several important pre-flight Monte Carlo analyses.

To avoid a nose-first reentry, it was critical that the BTMs induce a sufficient tumble rate that would persist as the FS flew through the wake of the USS. In addition, it was also necessary to accurately characterize the FS behavior in the wake region since that was also the time when far-field recontact was an issue. For this reason, the wake aerodynamic database that was discussed previously was developed and included in the POST2 reentry simulation.

The effect of including the wake database was assessed by comparing simulated results generated both with and without the wake aerodynamic model to the reconstructed flight data. A key result from this analysis is shown in Fig. 13 where the time history of the FS tumble rate (defined as the root-sum-square of the pitch and yaw body rates) from the BET is plotted for the first $30 \mathrm{~s}$ after separation. Also plotted are simulation results with and without wake aerodynamics. These results were from a post-flight simulation initiated at the BET separation state and utilized reconstructed atmosphere and RSRM thrust tailoff profiles. As seen in the plot, the simulated tumble rate profile matches the flight data much better when the wake aerodynamic model is included. The reason for this improved match is shown in the schematic drawing adjacent to the plot in Fig. 13. The firing of the BTMs cause the FS to rotate and translate. The rotation initially exposes the aft end of the FS to higher pressure flow outside of the wake which, in turn, reduces the tumble rate induced by the BTMs. As the FS begins to translate laterally, the aft end moves into the lower pressure of the wake while its forward end moves out of the wake and is into the higher pres- 

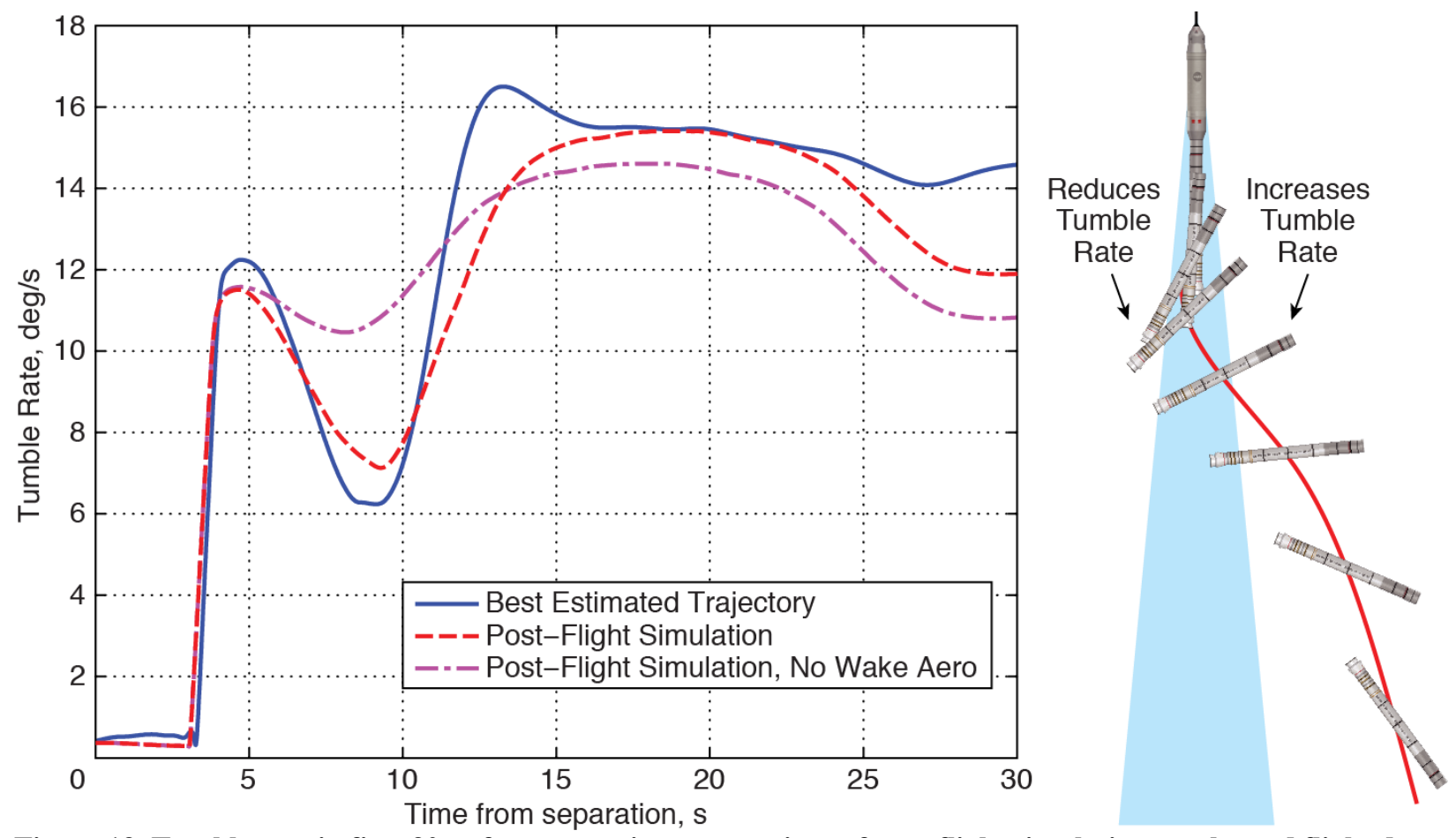

Figure 13. Tumble rate in first $30 \mathrm{~s}$ after separation, comparison of post-flight simulation results and flight data.

sure, thus tending to increase the tumble rate. By including the wake aerodynamic model, the dip in tumble rate observed during flight between 5 and 15 seconds after separation is reproduced in the simulation.

Another simulation result assessed after flight was the predicted FS reentry trim orientation. Reconstructed trajectory results indicated that the FS performed a tail-first reentry during flight, reaching a maximum dynamic pressure of 1,065 psf. In pre-flight reentry studies, a broad-side reentry was shown to be the most likely scenario; however, a tail-first reentry occurred in $37 \%$ of the off-nominal cases. The predicted trim orientation was most sensitive to the axial cg location and the location of the center of pressure. At broad-side attitudes during tumbling flight, the center of pressure is difficult to characterize. In addition, the FS cg can be affected by slag retained in the rear dome of the motor, which would tend to shift the cg rearward. Recall that the pre-flight Monte Carlo analysis was performed without slag to represent the worst-case scenario for nose first reentries. A second post-flight Monte Carlo analysis was also run assuming 2,000 $\mathrm{lb}$ of slag retention, to determine its effect on the percentage of cases with a particular trim orientation. The post-flight Monte Carlo also utilized the reconstructed separation state, atmosphere,

Table 3. Comparison of Pre-flight and Post-flight Monte Carlo Results with BET Flight Data at Maximum Dynamic Pressure.

\begin{tabular}{|c|c|c|c|c|c|c|c|}
\hline & \multicolumn{3}{|c|}{ Percentile } & \multicolumn{3}{|c|}{ Percentile } & \multirow[b]{2}{*}{ BET } \\
\hline & 0.13 & 50 & 99.87 & 0.13 & 50 & 99.87 & \\
\hline Max q, psf & 291.6 & 573.0 & 4420.4 & 327.3 & 675.2 & 3656.5 & 1064.8 \\
\hline Alpha Total, deg & 3.6 & 132.6 & 163.8 & 8.0 & 141.0 & 162.8 & 151.1 \\
\hline Mach & 1.71 & 2.47 & 3.62 & 1.75 & 2.40 & 3.32 & 2.51 \\
\hline Altitude, $\mathrm{ft}$ & 28850 & 62450 & 86098 & 32500 & 56467 & 83567 & 51986 \\
\hline Percent Nose-First & 4.1 & & & 1.0 & & & \\
\hline Percent Broad-Side & 58.6 & & & 46.5 & & & \\
\hline Percent Tail-First & 37.3 & & & 53.5 & & & \\
\hline
\end{tabular}

Pre-Flight Monte Carlo

Post-Flight Monte Carlo

Best Estimate Trajectory

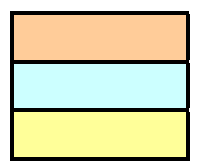


and RSRM thrust profile. Pre-flight and postflight statistics for total angle-of-attack, Mach number, and altitude at maximum dynamic pressure are given in Table 3 along with the values from the BET. The percentages of nosefirst, broad-side, and tail-first cases also given in Table 3 indicate that, when Monte Carlo assumptions were updated using post-flight knowledge, a tail-first reentry was the most likely reentry mode, which is consistent with the BET reentry.

Additional comparisons of the flight data to the pre-flight Monte Carlo predictions are plotted in Figs. 14 and 15. Monte Carlo cases are displayed in gray while the BET is plotted in red, and results are shown up until the time of parachute deployment. For consistency, only tail-first Monte Carlo cases were used in the comparisons. Figure 14 shows that the total angle-of-attack profile was within the Monte Carlo bounds for most of the reentry, including during max-q. After max-q, when the FS entered into a flat spin, the oscillations about 90 deg angle-ofattack observed during flight were slightly wider than the pre-flight Monte Carlo bounds, but were acceptable for parachute deployment. Figure 15 shows that the Mach vs dynamic pressure profile was well within the pre-flight Monte Carlo bounds.

\section{Range Safety Targets}

Using reconstructed trajectory data, the impact of both stages was compared to the preflight disposal footprints that were developed for range safety requirements.

Figure 16 shows the reconstructed Ares I-X ascent trajectory as well as the FS and USS reentry trajectories and corresponding splash down locations. For reference, the pre-flight disposal footprints delivered as part of the range safety package are also included in the figure. Since the FS was recovered, its impact location was known. However, during reentry the USS was only tracked using radar. Due to the eventual loss of radar contact, its location could only be determined down to an altitude of approximately 28,500 ft. Consequently, a postflight simulation that utilized state information from the radar data was employed to determine a projected splash down location. The final position of the FS and the projected final position of the USS are both marked in Fig. 16. The results show that both stages impacted near the center of their respective ellipses. The two stages were over 10 nmi apart and the USS splashed down nearly $40 \mathrm{~s}$ before the FS.

\section{Conclusion}

The Ares I-X Flight Test Vehicle was launched on October 28, 2009 and all major flight objectives, including those pertaining to stage separation and reentry, were successfully met. Post-flight analysis showed that no stage recontact occurred during separation, and the tumbling motion of the USS shortly after separation was similar to predicted behavior in pre-flight analyses. During flight, the FS exhibited atmospheric flight dynamics that compared well with pre-flight estimates, and it was possible to reasonably reproduce the flight behavior with small changes to 


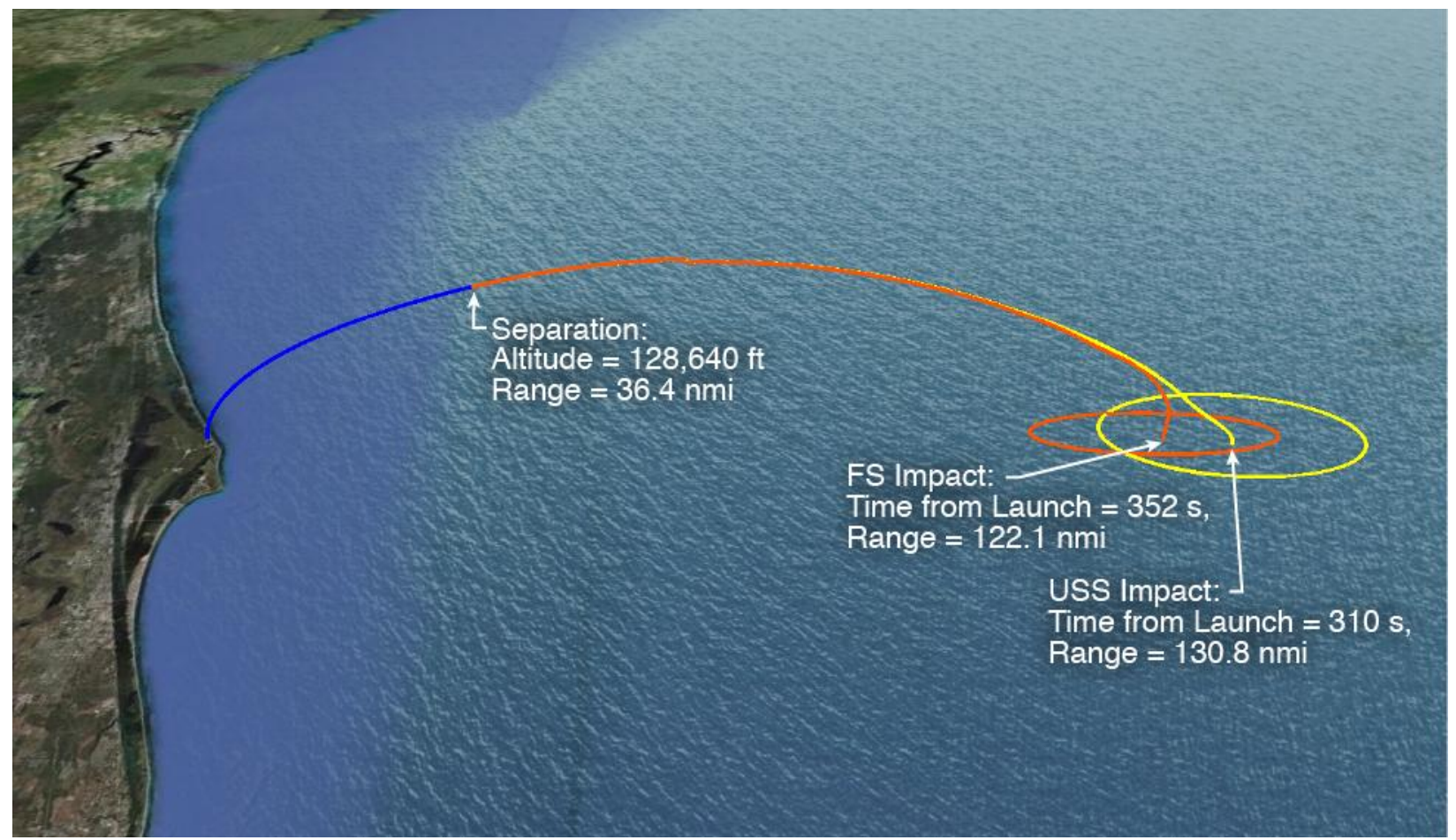

Figure 16. Ares I-X Trajectory showing the impact locations of the FS and USS compared to pre-flight disposal footprints.

simulation uncertainty models. The FS passed through peak dynamic pressure in a tail-first orientation, which had been shown to have a reasonable likelihood of occurring in pre-flight studies, and successfully reached parachute deployment conditions that were within the constraints of the recovery system. Both stages splashed down in the ocean well within their predicted impact footprints.

\section{Acknowledgments}

The authors wish to gratefully acknowledge Scott Angster who created the separation animations, Andre Brochier for his extensive work in simulation verification and Chris Karlgaard for trajectory reconstruction. The authors would also like to thank James Beaty for reviewing the paper and Anne Rhodes for creating the graphic art and preparing the final manuscript.

\section{References}

${ }^{1}$ Starr, B. R., Gumbert, C. R., and Tartabini, P. V., “Ares I-X Test Flight Reference Trajectory Development," 58th JANNAF Propulsion Meeting, April 2011.

${ }^{2}$ Smith, R. M., and Bryant, R. B., "Significant Technical Results for the Ares I-X Flight Test," 58th JANNAF Propulsion Meeting, April 2011.

${ }^{3}$ Striepe, S. A., Powell, R. W., Desai, P. N., Queen, E. M., Brauer, G. L., Cornick, D. E., Olson, D. W., Petersen, F. M., Stevenson, R., Engle, M. C., Marsh, S. M., and Gromko, A. M., "Program To Optimize Simulated Trajectories (POST II), Utilization Manual, Version 1.1. 6," Jan. 2004.

${ }^{4}$ Bauer, S. X., and Krist, S. E., "Aerodynamic Assessment of the Ares I-X Flight Test Vehicle," 58th JANNAF Propulsion Meeting, April 2011.

${ }^{5}$ Pinier, J. T., and Niskey, C. J., "Ares I and Ares I-X Stage Separation Aerodynamic Testing", 49th AIAA Aerospace Sciences Meeting and Exhibit, Orlando, FL, Jan. 2011, AIAA 2011-0169. (Invited)

${ }^{6}$ Purinton, D. C., Blevins, J. A., Pritchett, V., Haynes, D., and Carpenter, M., "Aerodynamic Characterization and Simulation of a Solid Rocket Booster During Reentry Flight,"

${ }^{7}$ Bonner, E., Clever, E., and Dunn, K., “Aerodynamic Preliminary Analysis System II, Part 1 - Theory,” NASA CR 182076, April 1991.

${ }^{8}$ Justus, C. G., and Leslie, F. W., "The NASA MSFC Earth Global Reference Atmospheric Model - 2007 Version,” NASA TM-2008-215581, Nov. 2008.

${ }^{9}$ King, R., Hengel, J. E., and Wolf, D., “Ares I First Stage Booster Deceleration System: An Overview,” AIAA 2009-2984, May 2009. 
${ }^{10}$ Starr, B. R., Gowan, J. W., and Thompson, B. G., “Ares I-X Range Safety Analyses Overview,” 58th JANNAF Propulsion Meeting, April 2011.

${ }^{11}$ Tarpley, A. F., Starr, B. R., Tartabini, P. V., Craig, A. S., Merry, C. M., Brewer, J. D., Davis, J. G., Dulski, M. B., Gimenez, A., Barron, M. K., "Ares I-X Range Safety Trajectory Analysis and IV\&V," 2011 AIAA Atmospheric Flight Mechanics Conference, AIAA, Reston, VA (submitted for publication).

${ }^{12}$ Hoerner, S. F., Fluid Dynamic Drag: Practical Information on Aerodynamic Drag and Hydrodynamic Resistance, Hoerner Fluid Dynamics, Albuquerque, 1965.

${ }^{13}$ Karlgaard, C. D., Beck, R. E., Starr, B. R., Derry, S. D., and Brandon, J., “Ares I-X Best Estimated Trajectory Analysis and Results," 58th JANNAF Propulsion Meeting, April 2011. 